\title{
Ação contra o câncer de pele em cidade com alto índice ultravioleta
}

\section{Action against skin cancer in a city with high ultraviolet index \\ Acción contra el cáncer de piel en ciudad con elevado índice de radiación ultravioleta}

\author{
José Paulo Ribeiro Júnior ii \\ Universidade Federal do Rio Grande do Norte - Natal (RN) - Brasil
}

Arnóbio da Penha Pacheco

Universidade Federal do Rio Grande do Norte - Natal (RN) - Brasil

Débora Dorneles Cunha de Queiroz Turíbio iD

Universidade Federal do Rio Grande do Norte - Natal (RN) - Brasil

Thaís Barros Felippe Jabour iD

Universidade Federal do Rio Grande do Norte - Natal (RN) - Brasil

\section{RESUMO}

Objetivo: Descrever a experiência de universitários no desenvolvimento de duas ações de conscientização sobre o câncer da pele. Síntese de dados: Os estudantes do curso de Medicina da Universidade Federal do Rio Grande do Norte, juntamente com médicos orientadores da Sociedade Brasileira de Dermatologia, elaboraram duas ações de conscientização para o combate ao câncer de pele associando medidas de educação em fotoproteção à aplicação de um questionário, visando ao planejamento futuro de ações mais direcionadas e eficazes. As ações ocorreram em duas praias durante o período de veraneio em 2020 . Os banhistas, após responderem às perguntas, receberam orientações a respeito dos hábitos saudáveis de fotoproteção de acordo com o Consenso Brasileiro de Fotoproteção. Conclusão: Os voluntários consideraram êxito no planejamento e execução da ação, com boa aceitação por parte dos banhistas e das entidades contribuintes.

Descritores: Neoplasias Cutâneas; Radiação Solar; Promoção da Saúde; Prevenção de Doenças.

\section{ABSTRACT}

Objective: To describe the experience of university students in developing two campaigns to fight skin cancer. Data synthesis: Medical students from Federal University of Rio Grande do Norte, along with supervising physicians from the Brazilian Society of Dermatology, developed two cancer-fighting interventions that associated photo-protection education measures with the application of a questionnaire for future planning of more precise interventions. The actions took place on two beaches during the summer period (2020) in order to reach a greater number of beachgoers. Beachgoers, after answering the questions, were instructed about healthy photoprotection habits according to the Brazilian Consensus on Photoprotection. Conclusion: The volunteers considered success in the planning and execution of the action, with good acceptance on the part of beachgoers and contributing entities.

Descriptors: Skin Neoplasms; Solar Radiation; Health Promotion; Disease Prevention.

\section{RESUMEN}

Objetivo: Describir la experiencia de universitarios para el desarrollo de dos acciones de concienciación sobre el cáncer de piel. Síntesis de datos: Los estudiantes del curso de Medicina de la Universidad Federal de Río Grande de Norte y los médicos tutores de la Sociedad Brasileña de Dermatología crearon dos acciones de concienciación para el combate del cáncer de piel asociando con medidas de educación en foto protección hasta la aplicación de una encuesta con el objetivo de planear acciones futuras más dirigidas y eficaces. Las acciones se dieron en dos playas durante el verano 2020. Tras contestar las preguntas, los bañistas recibieron orientaciones sobre los hábitos saludables de foto protección según el Consenso Brasileño de Foto Protección. Conclusión: Los voluntarios consideraron exitosos el planeamiento y la ejecución de la acción con buena aceptación de parte de los bañistas y de las entidades contribuyentes.

Descriptores: Neoplasias Cutáneas; Radiación Solar; Promoción de la Salud; Prevención de Enfermedades.

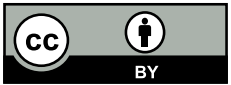




\section{INTRODUÇÃO}

Dividido nos subtipos melanoma e não melanoma, o câncer de pele é o câncer mais comum no mundo inteiro. A estimativa para o Brasil foi de que, no biênio 2018-2019, 165.580 novos casos de câncer da pele não-melanoma e 6.260 novos casos de melanoma foram diagnosticados ${ }^{(1)}$. Apesar dos números já serem alarmantes, o Instituto Nacional do Câncer (Inca) recomenda que as estimativas sejam consideradas como mínimas, tendo em vista a elevada probabilidade de sub-registro e sub-diagnóstico ${ }^{(2)}$.

É bem estabelecido que o excesso e cronicidade de exposição ao sol é o principal fator de risco para o surgimento do câncer de pele não melanoma ${ }^{(3,4)}$. Quanto ao melanoma, maior risco associa-se a histórico pessoal ou familiar de melanoma, além de exposição esporádica e intensa ao sol, com consequente queimadura solar, em mais de um episódio(3,5). A sensibilidade da pele ao sol, doenças imunossupressoras e exposição solar ocupacional são outros fatores de risco para todos os tipos de câncer de pele ${ }^{(6)}$.

A relação sol e câncer de pele é explicada pela agressão direta que a radiação ultravioleta causa na estrutura do DNA, provocando alterações estruturais e oxidação(7). Estudos mostram que Natal, capital do estado do Rio Grande do Norte, apresenta índices extremos de radiação solar em quase todos os dias do ano, significando necessidade de orientação à população para evitar exposição solar em qualquer horário do dia. Paradoxalmente, por se tratar de uma cidade com vasto litoral, o hábito de frequentar praia e veranear soma-se à exposição ocupacional do dia a dia, traduzindo uma realidade preocupante ${ }^{(8)}$.

Há evidência de que campanhas de conscientização sobre o tema causam impacto da infância à idade adulta nos hábitos de exposição à radiação ultravioleta (UV) e também na incidência do câncer de pele ${ }^{(9-11)}$.

Diante do exposto, o objetivo do presente estudo é descrever a experiência de universitários no desenvolvimento de duas ações de conscientização sobre o câncer de pele.

\section{SÍNTESE DOS DADOS}

O presente artigo trata-se de um relato de experiência resultante do trabalho de acadêmicos de Medicina da Liga Acadêmica de Dermatologia da Universidade Federal do Rio Grande do Norte (UFRN) em parceria com a Sociedade Brasileira de Dermatologia - Regional Rio Grande do Norte (SBD-RN) no desenvolvimento de ações de conscientização e combate ao câncer de pele e de conscientização de banhistas sobre hábitos saudáveis e risco de queimaduras, com o intuito de promover saúde e prevenir agravos, e, adicionalmente, elaboraram e aplicaram um questionário voltado para aferição dos hábitos de exposição solar e fotoproteção de acordo com a realidade brasileira.

Durante os meses de novembro e dezembro de 2019, realizaram-se pesquisas bibliográficas a respeito do impacto do câncer de pele e dos resultados das campanhas anuais para diagnóstico precoce de câncer de pele. A partir das plataformas de banco de dados SciElo, Lilacs, BVS e PubMed, pesquisaram-se os descritores "câncer de pele", "promoção da saúde”, "prevenção", "comportamento", "exposição solar", "fotoproteção" e "praia”, na língua portuguesa e inglesa, entre 2010 e 2020.

Encontros virtuais e presenciais para discussão de ideias a respeito da estrutura da campanha e para a elaboração de questionários para os banhistas aconteceram no mesmo período entre os acadêmicos de Medicina e o professor orientador. Inicialmente, percebeu-se que há uma quantidade mínima de trabalhos relatando campanhas voltadas à prevenção do câncer de pele (dois trabalhos), bem como levantamentos que apontem os hábitos de proteção solar cotidiano ou em momentos de exposição recreativa (nove trabalhos).

Entrevistas realizadas com banhistas em países europeus e na América Latina apontam que o uso de métodos de proteção solar é inadequado para a maior parte dos entrevistados, especialmente para os homens, havendo ainda desejo de parecer mais bronzeado, sendo frequentes as queimaduras solares. Todos esses estudos sugerem que é necessário que haja maior empenho na educação em saúde para difusão dos hábitos de prevenção de fotodano, sendo os banhistas um alvo estratégico(12-14).

Diante da imprescindibilidade de conhecer o perfil dos banhistas e seus hábitos, surgiu a necessidade de elaborar um questionário com perguntas baseadas nos fatores de risco para o câncer de pele e em hábitos preventivos. Não há na literatura a presença de um instrumento que atenda a essas necessidades na língua portuguesa. Por isso, desenvolveu-se um questionário com base no Beach Questionnaire, questionário espanhol validado para pesquisa sobre hábitos de exposição solar em banhistas ${ }^{(15)}$.

Optou-se pelo Beach Questionnaire devido à adequada seleção de perguntas, aplicáveis para avaliação do risco de desenvolvimento do câncer de pele, bem como pela forma como elas foram redigidas - diretas e de fácil 
interpretação ${ }^{(15)}$. O fato de não se tratar de um questionário extenso também contribuiu para a escolha, tornando-se aplicável à população em momentos de lazer ou descanso, sem maiores desconfortos. Além disso, para tornar-se mais verossímil à realidade local, formularam-se perguntas pertinentes sobre hábitos brasileiros, como o de veranear e de se bronzear para fins estéticos. Também se adicionaram algumas perguntas para a avaliação do grau de conhecimento da população sobre os temas exposição solar, câncer de pele e queimaduras solares. Utilizou-se o instrumento em alguns estudos ${ }^{(15,16)}$ e demonstrou ser uma ferramenta útil em pesquisas epidemiológicas ${ }^{(16)}$.

A primeira ação de prevenção contra o câncer de pele ocorreu no dia 18 de janeiro de 2020, das 8 às 12 horas, na praia de Pirangi, localizada na região metropolitana de Natal, Rio Grande do Norte. Escolheu-se a data por coincidir com um dia de eventos esportivos e de entretenimento realizado por uma revista local. A segunda ação aconteceu no dia 15 de fevereiro de 2020, das 8 às 12 horas, na praia da Redinha, zona norte de Natal, Rio Grande do Norte. Optou-se pela data porque, nesse dia, ocorreu o Festival da Ginga, evento gastronômico e cultural organizado pela prefeitura que atraiu vários banhistas para a praia.

Participaram das ações de conscientização dos banhistas 15 pessoas, entre elas o professor orientador e os acadêmicos de Medicina. A SBD-RN forneceu o transporte para o evento e os materiais para uso no dia, incluindo pranchetas, canetas, impressões de questionários e camisas padronizadas, oriundas da campanha Dezembro Laranja. A montagem de um estande possibilitou a conscientização sobre a necessidade do uso de protetores solares e a distribuição de algumas amostras, por doações de laboratórios farmacêuticos à SBD-RN.

Por decisão em conjunto, os participantes da ação se dividiram na praia para conversar individualmente com os banhistas a respeito dos hábitos de fotoproteção e fatores de risco para o câncer da pele, bem como para a aplicação do questionário. Após responderem ao questionário e receberem esclarecimentos sobre o tema, os banhistas se dirigiam ao estande de fotoproteção, onde eram apresentadas as diferentes opções de filtro solar e eram orientados sobre a forma de aplicação correta, necessidade de reaplicação periódica e particularidades para os diversos tipos de pele e idade.

No Brasil, pesquisas feitas com os trabalhadores de praias que sofriam com a exposição solar ocupacional, apesar de não terem englobado a população como um todo, já trouxeram informações importantes e que não podem ser ignoradas. Em um estudo realizado com pescadores no Pará, por exemplo, os resultados mostraram que $92 \%$ dos entrevistados não usavam protetor solar e mais da metade desconhecia que a radiação UV causava câncer, envelhecimento e manchas na pele ${ }^{(17)}$. Outro trabalho, com trabalhadores de uma praia urbana de Natal, mostrou índices um pouco melhores: $30 \%$ relatou usar protetor durante o trabalho e $24 \%$ afirmou não usar em momento algum ${ }^{(18)}$.

$\mathrm{Na}$ Espanha, em uma pesquisa concomitante com uma campanha com trabalhadores de um hotel à beiramar, os entrevistados apresentaram melhores hábitos de exposição solar, chegando a $71,3 \%$ de uso de filtro solar. Apesar de ainda não ser um número idealmente satisfatório, esse melhor percentual, comparado às pesquisas brasileiras, pode estar diretamente relacionado ao maior grau de instrução e escolaridade da população espanhola ${ }^{(19)}$.

O questionário desenvolvido para a ação descrita neste relato abordou como um dos temas a proteção solar diária, que, na maioria dos casos, só é realizada em momentos de exposição à praia ou piscina, com um maior cuidado com as crianças, sendo comum o uso de roupas de proteção UV, protetor solar e chapéu. Tal hábito não costumava se estender aos pais que, quando faziam, não seguiam todas as recomendações necessárias, tendo sido preciso reforçar a importância e o tempo de reaplicação do produto ${ }^{(20)}$.

Os estudantes observaram os principais receios do público das praias em relação à exposição solar, que relataram medo do câncer de pele ao mesmo tempo em que, paradoxalmente, referiam não possuir o hábito de observar os próprios sinais de pele. $O$ autoexame da pele e a prática da autopercepção do próprio corpo e suas alterações não fazem parte do costume dos brasileiros e impactam diretamente no diagnóstico precoce e prognóstico dos principais tipos de neoplasias cutâneas. Elucidando esse fato, uma pesquisa brasileira relatou a identificação mais precoce de câncer de pele não melanoma na Campanha Nacional de Prevenção ao Câncer de Pele quando comparado ao realizado pelas rotas convencionais do sistema público de saúde. Dos casos diagnosticados menores do que um centímetro de diâmetro, $86 \%$ vieram de campanhas e $71 \%$ dos referenciados pelo sistema convencional de saúde ${ }^{(21)}$.

$\mathrm{Na}$ Alemanha, percebeu-se um maior descuido da população no que tange ao autocuidado com as próprias lesões de pele. Após comparar campanhas de rastreamento de diferentes tipos de cânceres em mulheres, em se tratando do câncer de pele, ainda parecem não ter grande adesão, variando entre $0 \%$ e 3,7\% de acordo com o nível socioeconômico das mulheres entrevistadas ${ }^{(22)}$. Tendo em vista que campanhas para o diagnóstico precoce aumentam em $38 \%$ a chance de melanoma diagnosticado com 0,75 milímetros ou menos e reduzem consideravelmente a morte por essa causa ${ }^{(23)}$, é necessário criar estratégias para aumentar a adesão da população às campanhas de prevenção ao câncer de pele, dando atenção especial à com menor nível socioeconômico. 
Observou-se, a partir da aplicação do questionário durante a ação de prevenção, uma mudança de hábito dos banhistas no que diz respeito ao uso do bronzeador, que era comumente empregado nas últimas décadas. Endossando essa percepção, uma revisão sistemática prévia evidenciou aumento de $10 \%$ no uso de protetor solar e diminuição de $4 \%$ no uso de bronzeador. Neste trabalho, essa melhoria correlacionou-se aos efeitos obtidos por campanhas de combate ao câncer de pele em diferentes países ${ }^{(24)}$.

Durante a realização da ação e diante do consenso dos voluntários acerca da percepção de que a educação em saúde ainda precisa ser trabalhada no Brasil, chegou-se à discussão, entre o grupo, do impacto financeiro atual que esse problema representa e do quanto, em vidas e em números, uma melhor instrução populacional pode beneficiar o país. A exemplo, o governo australiano adotou uma estratégia preventiva permanente ${ }^{(23)}$. Nesse país, as neoplasias de pele correspondem a maior parcela dos gastos públicos entre todos os cânceres, apesar de ser apenas a vigésima causa de morte no país ${ }^{(25,26)}$. Segundo estudos locais, o Sun Smart, programa de prevenção realizado desde a década de 1980, resultará na redução de 120.000 anos de vida perdidos ajustados por incapacidade (DALYs) para o país em um período de 20 anos (2003-2022), somado à redução no uso dos serviços de saúde e economia de 2,30 dólares para cada dólar investido em campanhas ${ }^{(27)}$.

Outro estudo realizado na Austrália avaliou a relevância de campanhas de educação em massa no âmbito financeiro. Gastou-se 15,63 milhões de dólares com a campanha, com economia de 60,17 milhões de dólares em tratamentos para câncer de pele, tendo uma relação custo-benefício de $3,85^{(28)}$. Sendo assim, é realmente indiscutível o benefício em longo prazo em termos de saúde pública.

O presente trabalho trouxe também uma relevante visão sobre a necessidade de campanhas permanentes de combate ao câncer de pele em âmbito nacional. Sugere-se que uma ação anual pontual para diagnóstico do câncer, a despeito do que ocorre no Dia Nacional de Prevenção ao Câncer da Pele, mostra-se insuficiente para impactar de forma ampla a comunidade. Para tanto, faz-se necessário apoio financeiro de entidades públicas e privadas, capaz de proporcionar um local atrativo para aproximação da população. Canais como televisão, rádio, páginas de grande veiculação na internet e em redes sociais poderiam ser ferramentas para divulgação de informações importantes.

Como limitações à experiência vivenciada, tem-se o fato de ter sido realizada em apenas duas praias de um único estado brasileiro e de não conseguir avaliar o impacto das ações sobre a mudança de hábitos entre os banhistas, não sendo este o objeto da ação de prevenção. Entretanto, serviu como base para pesquisas e novas propostas de ação de conscientização contra o câncer de pele.

\section{CONCLUSÃO}

Considera-se que os universitários planejaram e executaram com o êxito a ação, desenvolvendo habilidades de criatividade, cooperação, comunicação e desenvolvimento estratégico. O questionário elaborado permite fácil coleta de informações e a interação com os banhistas contribuiu para conscientizar sobre a importância de medidas de prevenção do câncer da pele e avaliar o grau de conhecimento das pessoas, norteando o desenvolvimento de futuras campanhas.

\section{AGRADECIMENTOS E CONFLITOS DE INTERESSE}

Os autores agradecem o apoio da Sociedade Brasileira de Dermatologia - Regional Rio Grande do Norte (SBD-RN) para a realização da ação.

Os autores declaram que não houve conflitos de interesses na realização do trabalho.

\section{FONTES DE FINANCIAMENTO}

A Sociedade Brasileira de Dermatologia - Regional Rio Grande do Norte (SBD-RN) forneceu material e transporte para realização da ação que originou o estudo.

\section{CONTRIBUIÇÕES}

Todos os autores contribuíram com a elaboração e delineamento do estudo; a aquisição, análise e interpretação de dados; e a redação e/ou revisão do manuscrito. 


\section{REFERÊNCIAS}

1. Instituto Nacional do Câncer. Estimativa 2018: incidência de câncer no Brasil. Brasília: INCA; 2017.

2. Costa CS. Epidemiologia do câncer de pele no Brasil e evidências sobre sua prevenção. Diagn Tratamento. 2012;17(4):206-8.

3. Linares MA, Zakaria A, Nizran P. Skin Cancer. Prim Care. 2015;42(4):645-59.

4. Watson M, Holman DM, Maguire-Eisen M. Ultraviolet radiation exposure and its impact on skin cancer risk. Semin Oncol Nurs. 2016;32(3):241-54.

5. Carr S, Smith C, Wernberg J. Epidemiology and risk factors of melanoma. Surg Clin North Am. 2020;100(1):1-12.

6. Kütting B, Drexler H. UV-induced skin cancer at workplace and evidence-based prevention. Int Arch Occup Environ Health. 2010;23;83(8):843-54.

7. Kabir Y, Seidel R, McKnight B, Moy R. DNA Repair Enzymes: an important role in skin cancer prevention and reversal of photodamage- A Review of the Literature. J Drugs Dermatol. 2015;297-301.

8. Silva FC. Estudo da radiação ultravioleta na Cidade de Natal-RN [dissertação]. Natal: Universidade Federal do Rio Grande do Norte; 2008.

9. Thoonen K, Osch LV, Vries H, Jongen S, Schneider F. Are environmental interventions targeting skin cancer prevention among children and adolescents effective? a systematic review. Int J Environ Res Public Health. 2020;17(2):529.

10. Henrikson NB, Morrison CC, Blasi PR, Nguyen M, Shibuya KC, Patnode CD. Behavioral counseling for skin cancer prevention: a systematic evidence review for the U.S. Preventive Services Task Force. Rockville (MD): Agency for Healthcare Research and Quality (US); 2018.

11. Brunssen A, Waldmann A, Eisemann N, Katalinic A. Impact of skin cancer screening and secondary prevention campaigns on skin cancer incidence and mortality: a systematic review. J Am Acad Dermatol. 2017;76(1):129-39.

12. Martín MT, Sánchez ND, Sánchez NB, Tudela GO, Montero JCT, Mesa MLJ, et al. Skin cancer prevention campaign aimed at beachgoers on the Costa del Sol (southern Spain). Int J Dermatol. 2014;29;53(11):e526-30.

13. Haluza D, Simic $S$, Moshammer H. Sun exposure prevalence and associated skin health habits: results from the Austrian Population-Based UVSkinRisk Survey. Int J Environ Res Public Health. 2016;19;13(1):141.

14. Huamanchumo CJT, Muñoz SJB, Tineo LMV, Fernandez JP, Tineo OWV, Muñoz RMB, et al. Awareness, behavior and attitudes concerning sun exposure among beachgoers in the northern coast of Peru. PeerJ. 2019;15;7:e6189.

15. Martín MT, Sánchez NB, Ruiz FR, Canedo IF, Sandoval AR, Palliser JP, et al. Validation of a spanish questionnaire to evaluate habits, attitudes, and understanding of exposure to sunlight: the beach questionnaire. Actas Dermosifiliogr. 2009;100(7):586-95.

16. Morano TF, Martín MT, Ruiz FR, Sánchez NB, Eisman AB. Sensitivity to change of the Beach Questionnaire to behaviour, attitudes and knowledge related to sun exposure: quasi-experimental before-after study. BMC Public Health. 2015;15(1).

17. Rebelo EB, Oliveira KMR, Lobato TM, Silva WG, Bandeira CCA, Rocha RSB, et al. Exposição solar e envelhecimento precoce em trabalhadores praianos do município de Salinópolis/PA. Estud Interdiscip Envelhec. 2018;23:3:159-73.

18. Meyer PF, Silva RMV, Carvalho MGF, Nóbrega MM, Barreto AS, Aires FS, et al. Inquiry on the sun exposition in beach workers. Rev Bras Promoç Saúde. 2012;25(1):103-9.

19. Montero PG, Sánchez NB, Ruíz FR, Cayetano JFM, Canedo IF, Martín MT. Preventing Skin Cancer Among Staff and Guests at Seaside Hotels. J Cancer Educ. 2019.

20. Schalka S, Steiner D, Ravelli FN, Steiner T, Terena AC, Marçon CR, et al. Consenso Brasileiro de Fotoproteção. An Bras Dermatol. 2014;89(6):75. 
21. Wakiyama TP, França MLM, Carvalho LP, Marques MEA, Miot HA, Schmitt JV. Initial basal cell carcinomas diagnosed in the National Campaign for Skin Cancer Prevention are smaller than those identified by the conventional medical referral system. An Bras Dermatol. 2017;92(1):26-9.

22. Pezzotto SM, Nieto CS, Baroni MA, Iwanow P, Dagatti MS. Estudio acerca del conocimiento, actitudes y prácticas del cribado de cáncer de piel, mama y cérvix en una muestra de mujeres en Concordia, Argentina. Rev Fac Cienc Méd (Córdoba). 2019;76(1):11.

23. Sandhu PK, Elder R, Patel M, Saraiya M, Holman DM, Perna F, et al. Community-wide Interventions to Prevent Skin Cancer. Am J Prev Med. 2016;51(4):531-9.

24. Brunssen A, Waldmann A, Eisemann N, Katalinic A. Impact of skin cancer screening and secondary prevention campaigns on skin cancer incidence and mortality: a systematic review. J Am Acad Dermatol. 2017;76(1):129-39.

25. Cancer Council. National Skin Cancer Action Week 2019 [Internet]. 2019 [acesso em 2020 Jan 26]. Disponível em: https://www.cancer.org.au/preventing-cancer/sun-protection/campaigns-and-events/nationalskin-cancer-action-week.html

26. Cancer Council Australia. Skin cancer incidence and mortality [Internet]. 2020 [acesso em 2020 Jan 26]. Disponível em: https://wiki.cancer.org.au/skincancerstats/Skin_cancer_incidence_and_mortality

27. Australian Bureau of Statistics. Australia's leading causes of death, 2018 [Internet]. 2018 [acesso em 2020 Jan 26]. Disponível em: https://www.abs.gov.au/ausstats/abs@.nsf/mf/3303.0 28. S

28. Shih STF, Carter R, Sinclair C, Mihalopoulos C, Vos T. Economic evaluation of skin cancer prevention in Australia. Prev Med. 2009;49(5):449-53.

\author{
Endereço para correspondência \\ José Paulo Ribeiro Júnior \\ Hospital Universitário Onofre Lopes \\ Avenida Nilo Peçanha, 620 \\ Bairro: Petrópolis \\ CEP: 59012-300 - Natal - RN - Brasil \\ E-mail: josepaulojr04@gmail.com
}

Como citar: Ribeiro JP Júnior, Pacheco AP, Turíbio DDCQ, Jabour TBF. Ação contra o câncer de pele em cidade com alto índice ultravioleta. Rev Bras Promoç Saúde. 2020;33:10695. 